\title{
The Role of pH in Modulating the Electronic State Properties of Minocycline Drug and Its Inclusion within Micellar Carriers
}

\author{
Catia Clementi, Alessio Cesaretti, Benedetta Carlotti, Fausto Elisei
}

Department of Chemistry, Biology and Biotechnology and Center of Excellence on the Innovative Nanostructured Materials (CEMIN), University of Perugia, via Elce di Sotto 8, 06123 Perugia (Italy)

\section{Supporting information}

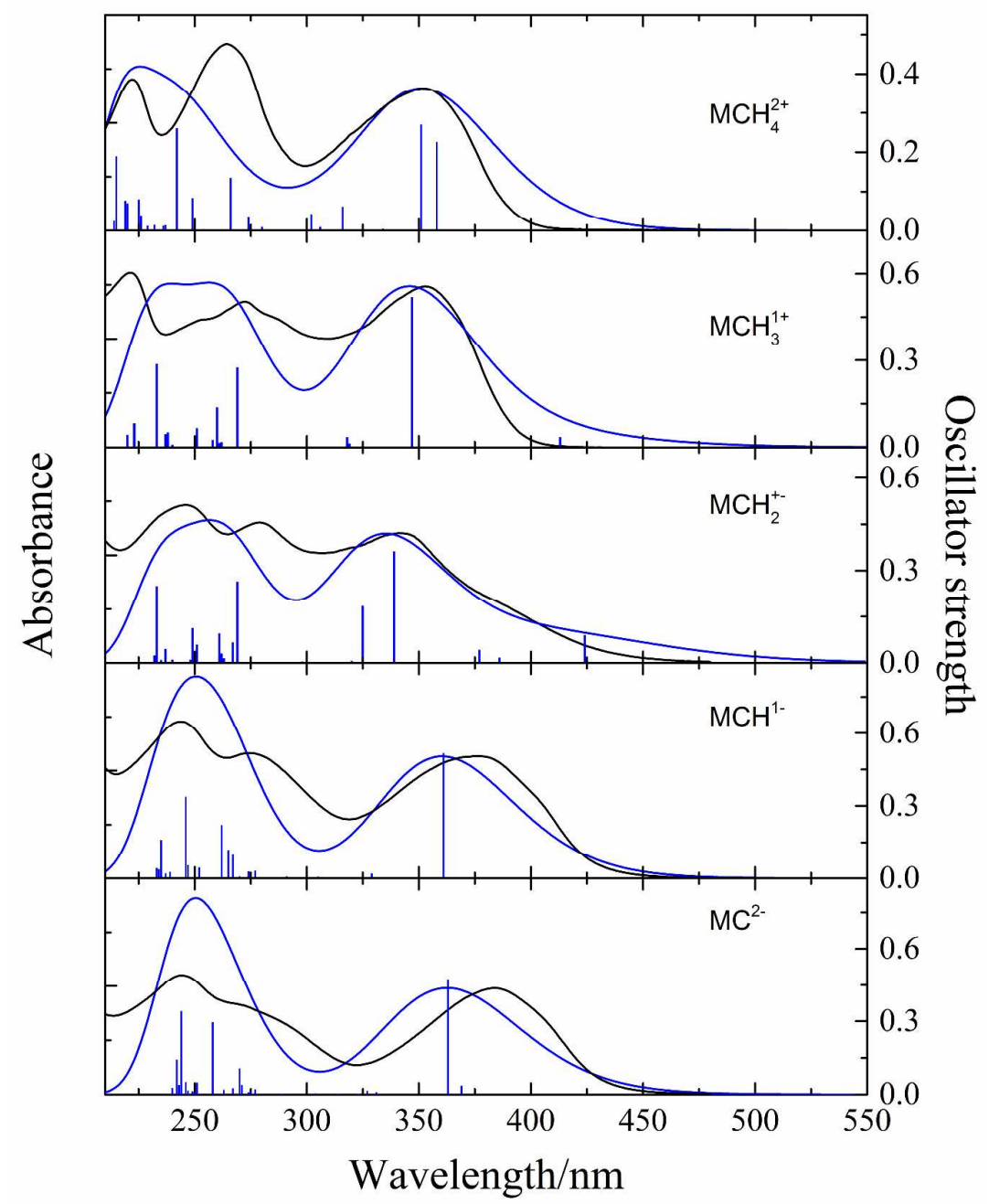

Figure S1. Absorption spectra and oscillator strengths of MC (blue lines and bars) calculated by TD-DFT theory, B3LYP functional, and $6-31 \mathrm{G}(\mathrm{d})$ basis set in water (simulated by the CPCM model), after geometrical optimization by use of the same functional and basis set. The experimental absorption spectra recorded in water solution (black lines) are also shown for comparison. 
Table S1. Experimental and theoretical absorption maxima of the protonated species of MC, main computed transition wavelengths and energies, oscillator strengths, and composition in terms of molecular orbitals obtained by the B3LYP/6-31G(d) model in water (CPCM).

\begin{tabular}{|c|c|c|c|c|c|c|}
\hline pH & species & $\exp . \max / \mathrm{nm}$ & th. $\max / \mathrm{nm}$ & th. trans/nm & $f$ & composition \\
\hline \multirow[t]{32}{*}{2.3} & $\mathrm{MCH}_{4}{ }^{2+}$ & 351 & 351 & $358\left(\mathrm{~S}_{1}\right)$ & 0.2247 & $\mathrm{H} \rightarrow \mathrm{L}(56 \%)$ \\
\hline & & & & & & $\mathrm{H}-1 \rightarrow \mathrm{L}+1(12 \%)$ \\
\hline & & & & & & $\mathrm{H} \rightarrow \mathrm{L}+1(12 \%)$ \\
\hline & & & & & & $\mathrm{H}-2 \rightarrow \mathrm{L}+1(11 \%)$ \\
\hline & & & & $351\left(\mathrm{~S}_{2}\right)$ & 0.2696 & $\mathrm{H} \rightarrow \mathrm{L}(41 \%)$ \\
\hline & & & & & & $\mathrm{H} \rightarrow \mathrm{L}+1(19 \%)$ \\
\hline & & & & & & $\mathrm{H}-2 \rightarrow \mathrm{L}+1(16 \%)$ \\
\hline & & & & & & $\mathrm{H}-1 \rightarrow \mathrm{L}+1(15 \%)$ \\
\hline & & 264 & $244^{\text {sh }}$ & $266\left(\mathrm{~S}_{10}\right)$ & 0.1339 & $\mathrm{H}-4 \rightarrow \mathrm{L}(53 \%)$ \\
\hline & & & & & & $\mathrm{H}-5 \rightarrow \mathrm{L}(29 \%)$ \\
\hline & & & & & & $\mathrm{H}-6 \rightarrow \mathrm{L}(10 \%)$ \\
\hline & & & & $249\left(\mathrm{~S}_{12}\right)$ & 0.0825 & $\mathrm{H}-3 \rightarrow \mathrm{L}+1(23 \%)$ \\
\hline & & & & & & $\mathrm{H} \rightarrow \mathrm{L}+2(14 \%)$ \\
\hline & & & & & & $\mathrm{H}-1 \rightarrow \mathrm{L}+2(10 \%)$ \\
\hline & & & & $242\left(\mathrm{~S}_{14}\right)$ & 0.2599 & $\mathrm{H}-5 \rightarrow \mathrm{L}+1(32 \%)$ \\
\hline & & & & & & $\mathrm{H}-6 \rightarrow \mathrm{L}+1(24 \%)$ \\
\hline & & & & & & $\mathrm{H}-5 \rightarrow \mathrm{L}+1(24 \%)$ \\
\hline & & 222 & 225 & $225\left(\mathrm{~S}_{20}\right)$ & 0.0666 & $\mathrm{H}-7 \rightarrow \mathrm{L}(50 \%)$ \\
\hline & & & & & & $\mathrm{H}-1 \rightarrow \mathrm{L}+2(16 \%)$ \\
\hline & & & & & & $\mathrm{H}-7 \rightarrow \mathrm{L}+1(15 \%)$ \\
\hline & & & & $225\left(\mathrm{~S}_{21}\right)$ & 0.0792 & $\mathrm{H} \rightarrow \mathrm{L}+3(15 \%)$ \\
\hline & & & & & & $\mathrm{H}-2 \rightarrow \mathrm{L}+3(13 \%)$ \\
\hline & & & & & & $\mathrm{H}-2 \rightarrow \mathrm{L}+3(11 \%)$ \\
\hline & & & & & & $\mathrm{H}-1 \rightarrow \mathrm{L}+3(10 \%)$ \\
\hline & & & & $220\left(\mathrm{~S}_{22}\right)$ & 0.0692 & $\mathrm{H} \rightarrow \mathrm{L}+4(70 \%)$ \\
\hline & & & & & & $\mathrm{H}-2 \rightarrow \mathrm{L}+2(13 \%)$ \\
\hline & & & & $219\left(\mathrm{~S}_{23}\right)$ & 0.0762 & $\mathrm{H}-8 \rightarrow \mathrm{L}(70 \%)$ \\
\hline & & & & & & $\mathrm{H} \rightarrow \mathrm{L}+4(9 \%)$ \\
\hline & & & & & & $\mathrm{H}-8 \rightarrow \mathrm{L}+1(8 \%)$ \\
\hline & & & & $215\left(\mathrm{~S}_{24}\right)$ & 0.1886 & $\mathrm{H}-2 \rightarrow \mathrm{L}+3(28 \%)$ \\
\hline & & & & & & $\mathrm{H}-2 \rightarrow \mathrm{L}+2(26 \%)$ \\
\hline & & & & & & $\mathrm{H}-1 \rightarrow \mathrm{L}+4(17 \%)$ \\
\hline \multirow[t]{5}{*}{3.9} & $\mathrm{MCH}_{3}{ }^{+}$ & 352 & 346 & $347\left(\mathrm{~S}_{3}\right)$ & 0.5183 & $\mathrm{H}-2 \rightarrow \mathrm{L}(94 \%)$ \\
\hline & & 271 & 257 & $269\left(\mathrm{~S}_{10}\right)$ & 0.2734 & $\mathrm{H}-2 \rightarrow \mathrm{L}(66 \%)$ \\
\hline & & & & & & $\mathrm{H}-1 \rightarrow \mathrm{L}+1(15 \%)$ \\
\hline & & 222 & 235 & $233\left(\mathrm{~S}_{22}\right)$ & 0.2850 & $\mathrm{H} \rightarrow \mathrm{L}+4(77 \%)$ \\
\hline & & & & & & $\mathrm{H}-3 \rightarrow \mathrm{L}+1(9 \%)$ \\
\hline \multirow[t]{11}{*}{6.4} & $\mathrm{MCH}_{2}{ }^{ \pm}$ & 342 & 335 & $339\left(\mathrm{~S}_{4}\right)$ & 0.3604 & $\mathrm{H}-3 \rightarrow \mathrm{L}(91 \%)$ \\
\hline & & & & $325\left(\mathrm{~S}_{5}\right)$ & 0.1825 & $\mathrm{H}-4 \rightarrow \mathrm{L}(89 \%)$ \\
\hline & & 280 & 257 & $269\left(S_{11}\right)$ & 0.2642 & $\mathrm{H}-1 \rightarrow \mathrm{L}+1(45 \%)$ \\
\hline & & & & & & $\mathrm{H}-2 \rightarrow \mathrm{L}+1(15 \%)$ \\
\hline & & & & & & $\mathrm{H}-7 \rightarrow \mathrm{L}(14 \%)$ \\
\hline & & & & $261\left(\mathrm{~S}_{15}\right)$ & 0.0940 & $\mathrm{H} \rightarrow \mathrm{L}+2(73 \%)$ \\
\hline & & & & & & $\mathrm{H}-7 \rightarrow \mathrm{L}(13 \%)$ \\
\hline & & & & $249\left(\mathrm{~S}_{18}\right)$ & 0.1110 & $\mathrm{H} \rightarrow \mathrm{L}+4(81 \%)$ \\
\hline & & 245 & $239^{\text {sh }}$ & $233\left(\mathrm{~S}_{23}\right)$ & 0.2488 & $\mathrm{H}-1 \rightarrow \mathrm{L}+3(57 \%)$ \\
\hline & & & & & & $\mathrm{H}-4 \rightarrow \mathrm{L}+1(9 \%)$ \\
\hline & & & & & & $\mathrm{H}-5 \rightarrow \mathrm{L}+1(8 \%)$ \\
\hline \multirow[t]{13}{*}{9.0} & $\mathrm{MCH}^{-}$ & 374 & 361 & $361\left(\mathrm{~S}_{1}\right)$ & 0.5145 & $\mathrm{H} \rightarrow \mathrm{L}(95 \%)$ \\
\hline & & 275 & \multirow{7}{*}{251} & $267\left(\mathrm{~S}_{13}\right)$ & 0.0957 & $\mathrm{H} \rightarrow \mathrm{L}+3(52 \%)$ \\
\hline & & & & & & $\mathrm{H} \rightarrow \mathrm{L}+2(27 \%)$ \\
\hline & & & & $265\left(\mathrm{~S}_{14}\right)$ & 0.1169 & $\mathrm{H} \rightarrow \mathrm{L}+2(38 \%)$ \\
\hline & & & & & & $\mathrm{H} \rightarrow \mathrm{L}+3(28 \%)$ \\
\hline & & & & $262\left(\mathrm{~S}_{15}\right)$ & 0.2192 & $\mathrm{H}-3 \rightarrow \mathrm{L}+1(50 \%)$ \\
\hline & & & & & & $\mathrm{H}-4 \rightarrow \mathrm{L}+1(28 \%)$ \\
\hline & & 244 & & $246\left(\mathrm{~S}_{18}\right)$ & 0.3366 & $\mathrm{H}-7 \rightarrow \mathrm{L}(36 \%)$ \\
\hline & & & & & & $\mathrm{H} \rightarrow \mathrm{L}+4(22 \%)$ \\
\hline & & & & & & $\mathrm{H}-6 \rightarrow \mathrm{L}(14 \%)$ \\
\hline & & & & $235\left(\mathrm{~S}_{23}\right)$ & 0.1594 & $\mathrm{H}-3 \rightarrow \mathrm{L}+2(38 \%)$ \\
\hline & & & & & & $\mathrm{H}-1 \rightarrow \mathrm{L}+2(21 \%)$ \\
\hline & & & & & & $\mathrm{H}-4 \rightarrow \mathrm{L}+2(8 \%)$ \\
\hline \multirow[t]{9}{*}{11.3} & $\mathrm{MC}^{2-}$ & 382 & 362 & $369\left(\mathrm{~S}_{1}\right)$ & 0.0340 & $\mathrm{H}-1 \rightarrow \mathrm{L}(66 \%)$ \\
\hline & & & & & & $\mathrm{H}-5 \rightarrow \mathrm{L}(20 \%)$ \\
\hline & & & & $363\left(\mathrm{~S}_{2}\right)$ & 0.4733 & $\mathrm{H} \rightarrow \mathrm{L}(89 \%)$ \\
\hline & & 271 & 250 & $270\left(\mathrm{~S}_{13}\right)$ & 0.1053 & $\mathrm{H} \rightarrow \mathrm{L}+2(56 \%)$ \\
\hline & & & & & & $\mathrm{H}-6 \rightarrow \mathrm{L}(19 \%)$ \\
\hline & & & & $258\left(\mathrm{~S}_{16}\right)$ & 0.2946 & $\mathrm{H}-2 \rightarrow \mathrm{L}+1(63 \%)$ \\
\hline & & & & & & $\mathrm{H}-4 \rightarrow \mathrm{L}+1(16 \%)$ \\
\hline & & 244 & & $244\left(\mathrm{~S}_{22}\right)$ & 0.3400 & $\mathrm{H}-3 \rightarrow \mathrm{L}+2(36 \%)$ \\
\hline & & & & & & $\mathrm{H} \rightarrow \mathrm{L}+4(19 \%)$ \\
\hline
\end{tabular}


Table S2. Theoretical absorption wavelengths of $\mathrm{MCH}_{4}{ }^{2+}$, oscillator strengths, nature and composition in terms of molecular orbitals obtained by the $\mathrm{B} 3 \mathrm{LYP} / 6-31 \mathrm{G}(\mathrm{d})$ model in water (CPCM), together with the experimental absorption maxima.

\begin{tabular}{|c|c|c|c|c|c|}
\hline transition & $\boldsymbol{\lambda}_{\text {th }} / \mathbf{n m}$ & $\mathbf{f}$ & $\mathbf{M O}$ & $\boldsymbol{c}_{\boldsymbol{i}}^{\mathbf{2}} / \boldsymbol{\%}$ & $\boldsymbol{\lambda}_{\text {exp }} / \mathbf{n m}$ \\
\hline $\mathrm{S}_{0} \rightarrow \mathrm{S}_{1}$ & 358 & 0.2247 & $\pi_{H} \rightarrow \pi_{L}^{*}$ & 56 & \\
\hline $\mathrm{S}_{0} \rightarrow \mathrm{S}_{2}$ & 351 & 0.2696 & $\pi_{H} \rightarrow \pi_{L}^{*}$ & 41 & 351 \\
\hline $\mathrm{S}_{0} \rightarrow \mathrm{S}_{3}$ & 334 & 0.0031 & $\pi_{H-1} \rightarrow \pi_{L}^{*}$ & 76 & \\
\hline $\mathrm{S}_{0} \rightarrow \mathrm{S}_{4}$ & 316 & 0.0607 & $\pi_{H-2} \rightarrow \pi_{L}^{*}$ & 53 & \\
\hline $\mathrm{S}_{0} \rightarrow \mathrm{S}_{5}$ & 306 & 0.0077 & $\pi_{H} \rightarrow \pi_{L+1}^{*}$ & 39 & \\
\hline $\mathrm{S}_{0} \rightarrow \mathrm{S}_{6}$ & 302 & 0.0420 & $\pi_{H-2} \rightarrow \pi_{L}^{*}$ & 27 & \\
\hline $\mathrm{S}_{0} \rightarrow \mathrm{S}_{7}$ & 282 & 0.0015 & $\pi_{H-1} \rightarrow \pi_{L+1}^{*}$ & 64 & \\
\hline $\mathrm{S}_{0} \rightarrow \mathrm{S}_{8}$ & 280 & 0.0078 & $\pi_{H-3} \rightarrow \pi_{L}^{*}$ & 97 & \\
\hline $\mathrm{S}_{0} \rightarrow \mathrm{S}_{9}$ & 274 & 0.0352 & $\pi_{H-3} \rightarrow \pi_{L+1}^{*}$ & 47 & \\
\hline $\mathrm{S}_{0} \rightarrow \mathrm{S}_{10}$ & 266 & 0.1339 & $\pi_{H-4} \rightarrow \pi_{L}^{*}$ & 53 & 264 \\
\hline $\mathrm{S}_{0} \rightarrow \mathrm{S}_{11}$ & 254 & 0.0021 & $n_{H-6} \rightarrow \pi_{L}^{*}$ & 59 & \\
\hline $\mathrm{S}_{0} \rightarrow \mathrm{S}_{12}$ & 249 & 0.0825 & $\pi_{H-3} \rightarrow \pi_{L+1}^{*}$ & 23 & \\
\hline $\mathrm{S}_{0} \rightarrow \mathrm{S}_{13}$ & 243 & 0.0001 & $\pi_{H-4} \rightarrow \pi_{L+1}^{*}$ & 64 & \\
\hline $\mathrm{S}_{0} \rightarrow \mathrm{S}_{14}$ & 242 & 0.2599 & $\pi_{H-5} \rightarrow \pi_{L+1}^{*}$ & 32 & \\
\hline $\mathrm{S}_{0} \rightarrow \mathrm{S}_{15}$ & 237 & 0.0125 & $\pi_{H} \rightarrow \pi_{L+2}^{*}$ & 29 & \\
\hline $\mathrm{S}_{0} \rightarrow \mathrm{S}_{16}$ & 236 & 0.0107 & $\pi_{H-5} \rightarrow \pi_{L+1}^{*}$ & 25 & \\
\hline $\mathrm{S}_{0} \rightarrow \mathrm{S}_{17}$ & 232 & 0.0131 & $n_{H-7} \rightarrow \pi_{L+1}^{*}$ & 63 & \\
\hline $\mathrm{S}_{0} \rightarrow \mathrm{S}_{18}$ & 229 & 0.0106 & $n_{H-7} \rightarrow \pi_{L}^{*}$ & 17 & \\
\hline $\mathrm{S}_{0} \rightarrow \mathrm{S}_{19}$ & 226 & 0.0380 & $\pi_{H} \rightarrow \pi_{L+3}^{*}$ & 16 & \\
\hline $\mathrm{S}_{0} \rightarrow \mathrm{S}_{20}$ & 225 & 0.0666 & $n_{H-7} \rightarrow \pi_{L}^{*}$ & 50 & \\
\hline $\mathrm{S}_{0} \rightarrow \mathrm{S}_{21}$ & 225 & 0.0792 & $\pi_{H} \rightarrow \pi_{L+3}^{*}$ & 15 & \\
\hline $\mathrm{S}_{0} \rightarrow \mathrm{S}_{22}$ & 220 & 0.0692 & $\pi_{H} \rightarrow \pi_{L+4}^{*}$ & 70 & \\
\hline $\mathrm{S}_{0} \rightarrow \mathrm{S}_{23}$ & 219 & 0.0762 & $n_{H-8} \rightarrow \pi_{L}^{*}$ & 70 & \\
\hline $\mathrm{S}_{0} \rightarrow \mathrm{S}_{24}$ & 215 & 0.1886 & $\pi_{H-2} \rightarrow \pi_{L+3}^{*}$ & 28 & 222 \\
\hline $\mathrm{S}_{0} \rightarrow \mathrm{S}_{25}$ & 214 & 0.0229 & $\pi_{H-1} \rightarrow \pi_{L+3}^{*}$ & 55 & \\
\hline & & & & & \\
\hline
\end{tabular}




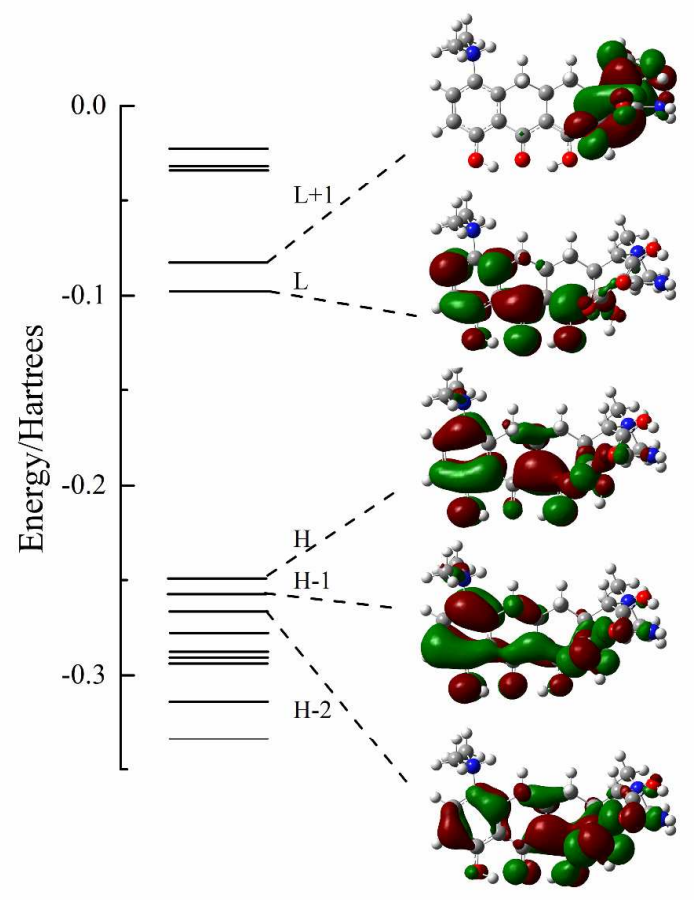

Figure S2. Molecular orbital energies and isodensity plots of $\mathrm{MCH}_{4}{ }^{2+}$ obtained by the B3LYP/6-31G(d) model.
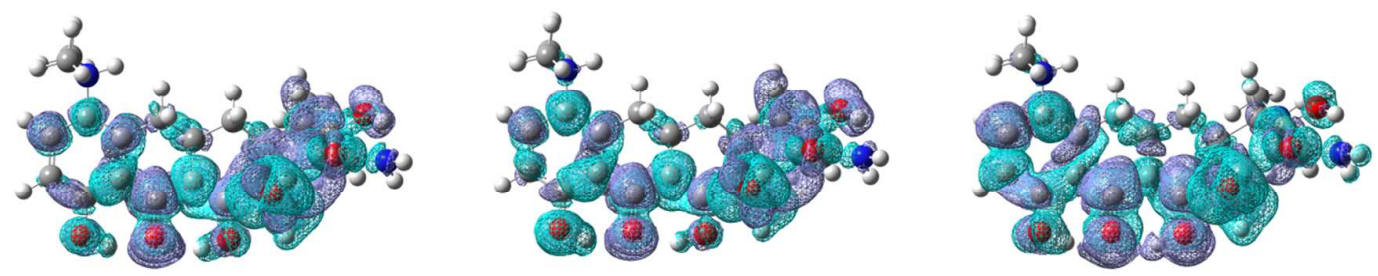

Figure S3. Effect of the $\mathrm{S}_{0} \rightarrow \mathrm{S}_{1}$ (left), $\mathrm{S}_{0} \rightarrow \mathrm{S}_{2}$ (center), and $\mathrm{S}_{0} \rightarrow \mathrm{S}_{3}$ (right) transitions on the electron density of $\mathrm{MCH}_{4}{ }^{2+}$ (increase and decrease of electron densities are represented by violet and cyan, respectively). 
Table S3. Theoretical absorption wavelengths of $\mathrm{MCH}_{3}{ }^{+}$, oscillator strengths, nature and composition in terms of molecular orbitals obtained by the B3LYP/6-31G(d) model in water (CPCM), together with the experimental absorption maxima.

\begin{tabular}{|c|c|c|c|c|c|}
\hline transition & $\lambda_{\text {th }} / \mathbf{n m}$ & $\mathbf{f}$ & $\mathbf{M O}$ & $\boldsymbol{c}_{\boldsymbol{i}}^{\mathbf{2}} / \mathbf{\%}$ & $\boldsymbol{\lambda}_{\text {exp }} / \mathbf{n m}$ \\
\hline $\mathrm{S}_{0} \rightarrow \mathrm{S}_{1}$ & 421 & 0.0015 & $\pi_{H} \rightarrow \pi_{L}^{*}$ & 65 & \\
\hline $\mathrm{S}_{0} \rightarrow \mathrm{S}_{2}$ & 413 & 0.0343 & $n_{H-1} \rightarrow \pi_{L}^{*}$ & 64 & \\
\hline $\mathrm{S}_{0} \rightarrow \mathrm{S}_{3}$ & 347 & 0.5183 & $\pi_{H-2} \rightarrow \pi_{L}^{*}$ & 94 & 352 \\
\hline $\mathrm{S}_{0} \rightarrow \mathrm{S}_{4}$ & 324 & 0.0020 & $\pi_{H-3} \rightarrow \pi_{L}^{*}$ & 62 & \\
\hline $\mathrm{S}_{0} \rightarrow \mathrm{S}_{5}$ & 319 & 0.0125 & $n_{H-1} \rightarrow \pi_{L+1}^{*}$ & 55 & \\
\hline $\mathrm{S}_{0} \rightarrow \mathrm{S}_{6}$ & 318 & 0.0344 & $\pi_{H-5} \rightarrow \pi_{L}^{*}$ & 64 & \\
\hline $\mathrm{S}_{0} \rightarrow \mathrm{S}_{7}$ & 311 & 0.0019 & $\pi_{H-7} \rightarrow \pi_{L}^{*}$ & 50 & \\
\hline $\mathrm{S}_{0} \rightarrow \mathrm{S}_{8}$ & 307 & 0.0021 & $\pi_{H-4} \rightarrow \pi_{L}^{*}$ & 71 & \\
\hline $\mathrm{S}_{0} \rightarrow \mathrm{S}_{9}$ & 280 & 0.0019 & $n_{H-6} \rightarrow \pi_{L}^{*}$ & 91 & \\
\hline $\mathrm{S}_{0} \rightarrow \mathrm{S}_{10}$ & 269 & 0.2734 & $\pi_{H} \rightarrow \pi_{L+1}^{*}$ & 66 & 271 \\
\hline $\mathrm{S}_{0} \rightarrow \mathrm{S}_{11}$ & 262 & 0.0175 & $n_{H-1} \rightarrow \pi_{L+4}^{*}$ & 31 & \\
\hline $\mathrm{S}_{0} \rightarrow \mathrm{S}_{12}$ & 261 & 0.0133 & $\pi_{H} \rightarrow \pi_{L+2}^{*}$ & 58 & \\
\hline $\mathrm{S}_{0} \rightarrow \mathrm{S}_{13}$ & 260 & 0.1368 & $\pi_{H-8} \rightarrow \pi_{L}^{*}$ & 52 & \\
\hline $\mathrm{S}_{0} \rightarrow \mathrm{S}_{14}$ & 258 & 0.0247 & $n_{H-1} \rightarrow \pi_{L+2}^{*}$ & 67 & \\
\hline $\mathrm{S}_{0} \rightarrow \mathrm{S}_{15}$ & 251 & 0.0651 & $\pi_{H-3} \rightarrow \pi_{L+1}^{*}$ & 32 & \\
\hline $\mathrm{S}_{0} \rightarrow \mathrm{S}_{16}$ & 248 & 0.0009 & $\pi_{H} \rightarrow \pi_{L+3}^{*}$ & 86 & \\
\hline $\mathrm{S}_{0} \rightarrow \mathrm{S}_{17}$ & 245 & 0.0020 & $n_{H-1} \rightarrow \pi_{L+3}^{*}$ & 81 & \\
\hline $\mathrm{S}_{0} \rightarrow \mathrm{S}_{18}$ & 242 & 0.0018 & $\pi_{H-2} \rightarrow \pi_{L+1}^{*}$ & 40 & \\
\hline $\mathrm{S}_{0} \rightarrow \mathrm{S}_{19}$ & 240 & 0.0077 & $\pi_{H-2} \rightarrow \pi_{L+1}^{*}$ & 42 & \\
\hline $\mathrm{S}_{0} \rightarrow \mathrm{S}_{20}$ & 238 & 0.0503 & $n_{H-6} \rightarrow \pi_{L+1}^{*}$ & 26 & \\
\hline $\mathrm{S}_{0} \rightarrow \mathrm{S}_{21}$ & 237 & 0.0447 & $\pi_{H-2} \rightarrow \pi_{L+2}^{*}$ & 78 & \\
\hline $\mathrm{S}_{0} \rightarrow \mathrm{S}_{22}$ & 233 & 0.2850 & $\pi_{H} \rightarrow \pi_{L+4}^{*}$ & 77 & \\
\hline $\mathrm{S}_{0} \rightarrow \mathrm{S}_{23}$ & 233 & 0.0105 & $n_{H-9} \rightarrow \pi_{L}^{*}$ & 94 & \\
\hline $\mathrm{S}_{0} \rightarrow \mathrm{S}_{24}$ & 223 & 0.0817 & $\pi_{H-5} \rightarrow \pi_{L+2}^{*}$ & 39 & \\
\hline $\mathrm{S}_{0} \rightarrow \mathrm{S}_{25}$ & 220 & 0.0415 & $\pi_{H-3} \rightarrow \pi_{L+2}^{*}$ & 53 & \\
\hline & & & & & \\
\hline
\end{tabular}




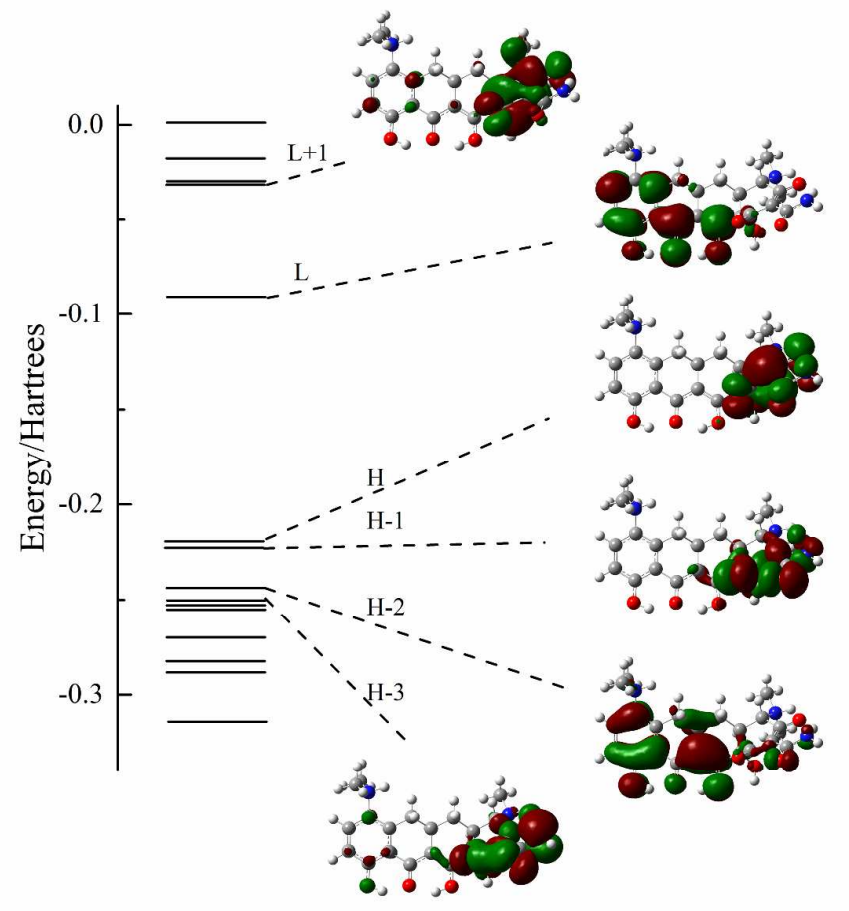

Figure S4. Molecular orbital energies and isodensity plots of $\mathrm{MCH}_{3}{ }^{+}$obtained by the $\mathrm{B} 3 \mathrm{LYP} / 6-31 \mathrm{G}(\mathrm{d})$ model.

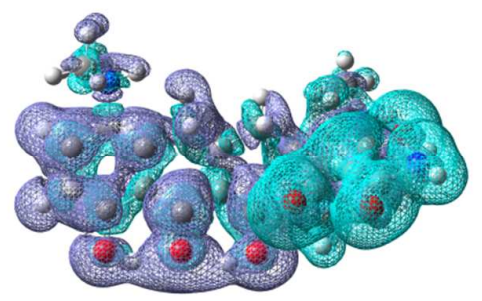

Figure S5. Effect of the $\mathrm{S}_{0} \rightarrow \mathrm{S}_{1}$ transition on the electron density of $\mathrm{MCH}_{3}{ }^{+}$(increase and decrease of electron densities are represented by violet and cyan, respectively). 
Table S4. Theoretical absorption wavelengths of $\mathrm{MCH}_{2}{ }^{ \pm}$, oscillator strengths, nature and composition in terms of molecular orbitals obtained by B3LYP/6-31G(d) model in water (CPCM), together with the experimental absorption maxima.

\begin{tabular}{|c|c|c|c|c|c|}
\hline transition & $\lambda_{\text {th }} / \mathbf{n m}$ & $\mathbf{f}$ & $\mathbf{M O}$ & $\boldsymbol{c}_{\boldsymbol{i}}^{\mathbf{2}} / \mathbf{\%}$ & $\boldsymbol{\lambda}_{\text {exp }} / \mathbf{n m}$ \\
\hline $\mathrm{S}_{0} \rightarrow \mathrm{S}_{1}$ & 424 & 0.0889 & $\pi_{H} \rightarrow \pi_{L}^{*}$ & 99 & $400_{\text {sh }}$ \\
\hline $\mathrm{S}_{0} \rightarrow \mathrm{S}_{2}$ & 386 & 0.0154 & $\pi_{H-2} \rightarrow \pi_{L}^{*}$ & 50 & \\
\hline $\mathrm{S}_{0} \rightarrow \mathrm{S}_{3}$ & 377 & 0.0406 & $\pi_{H-1} \rightarrow \pi_{L}^{*}$ & 52 & \\
\hline $\mathrm{S}_{0} \rightarrow \mathrm{S}_{4}$ & 339 & 0.3604 & $\pi_{H-3} \rightarrow \pi_{L}^{*}$ & 91 & 342 \\
\hline $\mathrm{S}_{0} \rightarrow \mathrm{S}_{5}$ & 325 & 0.1825 & $\pi_{H-4} \rightarrow \pi_{L}^{*}$ & 89 & \\
\hline $\mathrm{S}_{0} \rightarrow \mathrm{S}_{6}$ & 320 & 0.0053 & $\pi_{H-2} \rightarrow \pi_{L+1}^{*}$ & 71 & \\
\hline $\mathrm{S}_{0} \rightarrow \mathrm{S}_{7}$ & 310 & 0.0012 & $n_{H-9} \rightarrow \pi_{L}^{*}$ & 62 & \\
\hline $\mathrm{S}_{0} \rightarrow \mathrm{S}_{8}$ & 298 & 0.0031 & $n_{H-5} \rightarrow \pi_{L}^{*}$ & 76 & \\
\hline $\mathrm{S}_{0} \rightarrow \mathrm{S}_{9}$ & 285 & 0.0007 & $n_{H-6} \rightarrow \pi_{L}^{*}$ & 88 & \\
\hline $\mathrm{S}_{0} \rightarrow \mathrm{S}_{10}$ & 276 & 0.0010 & $\pi_{H} \rightarrow \pi_{L+1}^{*}$ & 98 & \\
\hline $\mathrm{S}_{0} \rightarrow \mathrm{S}_{11}$ & 269 & 0.2642 & $\pi_{H-1} \rightarrow \pi_{L+1}^{*}$ & 45 & 280 \\
\hline $\mathrm{S}_{0} \rightarrow \mathrm{S}_{12}$ & 267 & 0.0654 & $n_{H-7} \rightarrow \pi_{L}^{*}$ & 43 & \\
\hline $\mathrm{S}_{0} \rightarrow \mathrm{S}_{13}$ & 263 & 0.0138 & $n_{H-8} \rightarrow \pi_{L}^{*}$ & 77 & \\
\hline $\mathrm{S}_{0} \rightarrow \mathrm{S}_{14}$ & 262 & 0.0292 & $\pi_{H-2} \rightarrow \pi_{L+3}^{*}$ & 52 & \\
\hline $\mathrm{S}_{0} \rightarrow \mathrm{S}_{15}$ & 261 & 0.0940 & $\pi_{H} \rightarrow \pi_{L+2}^{*}$ & 73 & \\
\hline $\mathrm{S}_{0} \rightarrow \mathrm{S}_{16}$ & 251 & 0.0582 & $n_{H-5} \rightarrow \pi_{L+1}^{*}$ & 37 & \\
\hline $\mathrm{S}_{0} \rightarrow \mathrm{S}_{17}$ & 248 & 0.0092 & $\pi_{H-3} \rightarrow \pi_{L+1}^{*}$ & 69 & \\
\hline $\mathrm{S}_{0} \rightarrow \mathrm{S}_{18}$ & 249 & 0.1110 & $\pi_{H} \rightarrow \pi_{L+4}^{*}$ & 81 & \\
\hline $\mathrm{S}_{0} \rightarrow \mathrm{S}_{19}$ & 240 & 0.0094 & $n_{H-6} \rightarrow \pi_{L+1}^{*}$ & 34 & \\
\hline $\mathrm{S}_{0} \rightarrow \mathrm{S}_{20}$ & 237 & 0.0446 & $\pi_{H-1} \rightarrow \pi_{L+2}^{*}$ & 46 & \\
\hline $\mathrm{S}_{0} \rightarrow \mathrm{S}_{21}$ & 237 & 0.0155 & $n_{H-6} \rightarrow \pi_{L+1}^{*}$ & 29 & \\
\hline $\mathrm{S}_{0} \rightarrow \mathrm{S}_{22}$ & 235 & 0.0080 & $\pi_{H-2} \rightarrow \pi_{L+2}^{*}$ & 64 & \\
\hline $\mathrm{S}_{0} \rightarrow \mathrm{S}_{23}$ & 233 & 0.2488 & $\pi_{H-1} \rightarrow \pi_{L+3}^{*}$ & 57 & 245 \\
\hline $\mathrm{S}_{0} \rightarrow \mathrm{S}_{24}$ & 232 & 0.0230 & $\pi_{H-4} \rightarrow \pi_{L+1}^{*}$ & 67 & \\
\hline $\mathrm{S}_{0} \rightarrow \mathrm{S}_{25}$ & 229 & 0.0008 & $\pi_{H} \rightarrow \pi_{L+3}^{*}$ & 97 & \\
\hline & & & & & \\
\hline
\end{tabular}

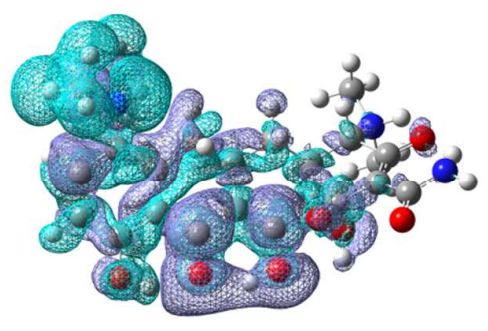

Figure S6. Effect of the $\mathrm{S}_{0} \rightarrow \mathrm{S}_{1}$ transition on the electron density of $\mathrm{MCH}_{2}^{ \pm}$(increase and decrease of electron densities are represented by violet and cyan, respectively). 
Table S5. Theoretical absorption wavelengths of $\mathrm{MCH}^{-}$, oscillator strengths, nature and composition in terms of molecular orbitals obtained by B3LYP/6-31G(d) model in water (CPCM), together with the experimental absorption maxima.

\begin{tabular}{|c|c|c|c|c|c|}
\hline transition & $\lambda_{\text {th }} / \mathbf{n m}$ & $\mathbf{f}$ & $\mathbf{M O}$ & $\boldsymbol{c}_{\boldsymbol{i}}^{\mathbf{2}} / \boldsymbol{\%}$ & $\boldsymbol{\lambda}_{\text {exp }} / \mathbf{n m}$ \\
\hline $\mathrm{S}_{0} \rightarrow \mathrm{S}_{1}$ & 361 & 0.5145 & $\pi_{H} \rightarrow \pi_{L}^{*}$ & 95 & 374 \\
\hline $\mathrm{S}_{0} \rightarrow \mathrm{S}_{2}$ & 355 & 0.0015 & $n_{H-2} \rightarrow \pi_{L}^{*}$ & 48 & \\
\hline $\mathrm{S}_{0} \rightarrow \mathrm{S}_{3}$ & 333 & 0.0023 & $\pi_{H} \rightarrow \pi_{L+1}^{*}$ & 40 & \\
\hline $\mathrm{S}_{0} \rightarrow \mathrm{S}_{4}$ & 329 & 0.0173 & $\pi_{H-1} \rightarrow \pi_{L}^{*}$ & 70 & \\
\hline $\mathrm{S}_{0} \rightarrow \mathrm{S}_{5}$ & 325 & 0.0044 & $\pi_{H} \rightarrow \pi_{L+1}^{*}$ & 53 & \\
\hline $\mathrm{S}_{0} \rightarrow \mathrm{S}_{6}$ & 305 & 0.0046 & $n_{H-4} \rightarrow \pi_{L}^{*}$ & 37 & \\
\hline $\mathrm{S}_{0} \rightarrow \mathrm{S}_{7}$ & 291 & 0.0053 & $\pi_{H-3} \rightarrow \pi_{L}^{*}$ & 68 & \\
\hline $\mathrm{S}_{0} \rightarrow \mathrm{S}_{8}$ & 282 & 0.0008 & $\pi_{H-1} \rightarrow \pi_{L+1}^{*}$ & 91 & \\
\hline $\mathrm{S}_{0} \rightarrow \mathrm{S}_{9}$ & 277 & 0.0293 & $\pi_{H-5} \rightarrow \pi_{L}^{*}$ & 74 & 275 \\
\hline $\mathrm{S}_{0} \rightarrow \mathrm{S}_{10}$ & 274 & 0.0265 & $n_{H-4} \rightarrow \pi_{L+1}^{*}$ & 35 & \\
\hline $\mathrm{S}_{0} \rightarrow \mathrm{S}_{11}$ & 270 & 0.0068 & $n_{H-2} \rightarrow \pi_{L+2}^{*}$ & 34 & \\
\hline $\mathrm{S}_{0} \rightarrow \mathrm{S}_{12}$ & 268 & 0.0023 & $\pi_{H-8} \rightarrow \pi_{L}^{*}$ & 37 & \\
\hline $\mathrm{S}_{0} \rightarrow \mathrm{S}_{13}$ & 267 & 0.0957 & $\pi_{H} \rightarrow \pi_{L+3}^{*}$ & 52 & \\
\hline $\mathrm{S}_{0} \rightarrow \mathrm{S}_{14}$ & 265 & 0.1169 & $\pi_{H} \rightarrow \pi_{L+2}^{*}$ & 38 & \\
\hline $\mathrm{S}_{0} \rightarrow \mathrm{S}_{15}$ & 262 & 0.2192 & $\pi_{H-3} \rightarrow \pi_{L+1}^{*}$ & 50 & \\
\hline $\mathrm{S}_{0} \rightarrow \mathrm{S}_{16}$ & 252 & 0.0432 & $\pi_{H-6} \rightarrow \pi_{L+1}^{*}$ & 32 & \\
\hline $\mathrm{S}_{0} \rightarrow \mathrm{S}_{17}$ & 247 & 0.0533 & $\pi_{H-1} \rightarrow \pi_{L+3}^{*}$ & 64 & \\
\hline $\mathrm{S}_{0} \rightarrow \mathrm{S}_{18}$ & 246 & 0.3366 & $\pi_{H-7} \rightarrow \pi_{L}^{*}$ & 36 & 244 \\
\hline $\mathrm{S}_{0} \rightarrow \mathrm{S}_{19}$ & 242 & 0.0008 & $\pi_{H-6} \rightarrow \pi_{L}^{*}$ & 50 & \\
\hline $\mathrm{S}_{0} \rightarrow \mathrm{S}_{20}$ & 239 & 0.0246 & $n_{H-10} \rightarrow \pi_{L+1}^{*}$ & 22 & \\
\hline $\mathrm{S}_{0} \rightarrow \mathrm{S}_{21}$ & 237 & 0.0186 & $n_{H-2} \rightarrow \pi_{L+3}^{*}$ & 49 & \\
\hline $\mathrm{S}_{0} \rightarrow \mathrm{S}_{22}$ & 234 & 0.0358 & $\pi_{H-5} \rightarrow \pi_{L+1}^{*}$ & 64 & \\
\hline $\mathrm{S}_{0} \rightarrow \mathrm{S}_{23}$ & 235 & 0.1594 & $\pi_{H-3} \rightarrow \pi_{L+2}^{*}$ & 38 & \\
\hline $\mathrm{S}_{0} \rightarrow \mathrm{S}_{24}$ & 234 & 0.0067 & $\pi_{H-1} \rightarrow \pi_{L+2}^{*}$ & 41 & \\
\hline $\mathrm{S}_{0} \rightarrow \mathrm{S}_{25}$ & 233 & 0.0402 & $\pi_{H} \rightarrow \pi_{L+4}^{*}$ & 38 & \\
\hline & & & & & \\
\hline
\end{tabular}




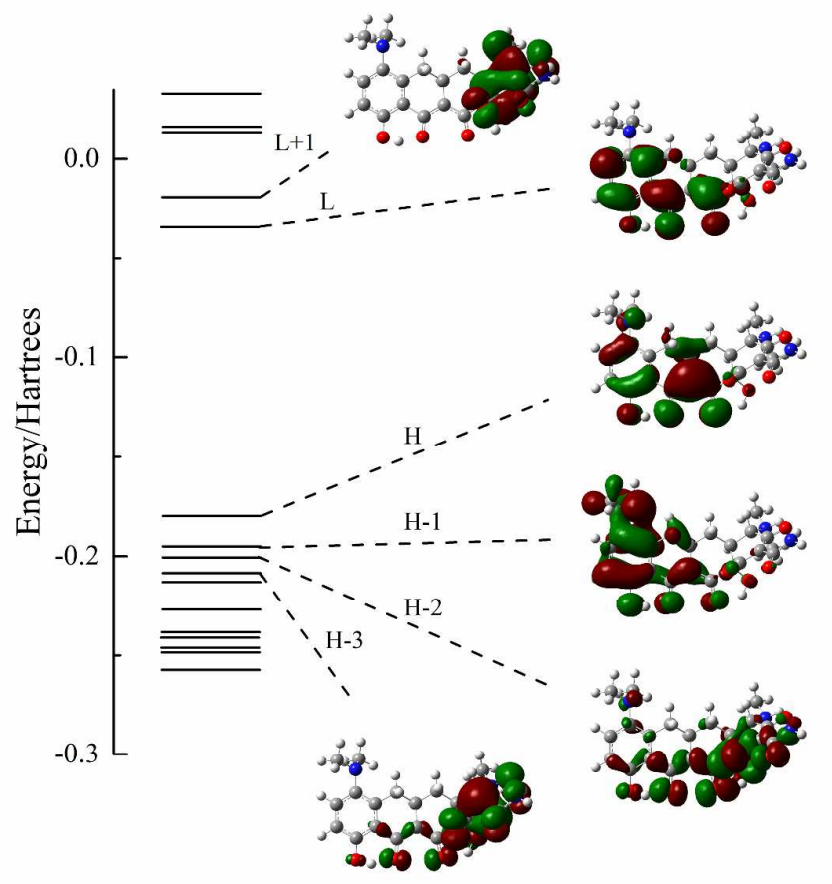

Figure S7. Molecular orbital energies and isodensity plots of $\mathrm{MCH}^{-}$obtained by the B3LYP/6-31G(d) model.

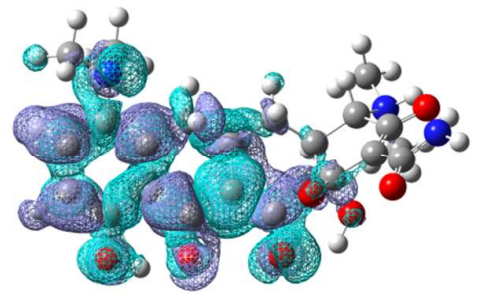

Figure S8. Effect of the $\mathrm{S}_{0} \rightarrow \mathrm{S}_{1}$ transition on the electron density of $\mathrm{MCH}^{-}$(increase and decrease of electron densities are represented by violet and cyan, respectively). 
Table S6. Theoretical absorption wavelengths of $\mathrm{MC}^{2-}$, oscillator strengths, nature and composition in terms of molecular orbitals obtained by B3LYP/6-31G(d) model in water (CPCM), together with the experimental absorption maxima.

\begin{tabular}{|c|c|c|c|c|c|}
\hline transition & $\lambda_{\text {th }} / \mathbf{n m}$ & $\mathbf{f}$ & $\mathbf{M O}$ & $\boldsymbol{c}_{\boldsymbol{i}}^{\mathbf{2}} / \mathbf{\%}$ & $\boldsymbol{\lambda}_{\text {exp }} / \mathbf{n m}$ \\
\hline $\mathrm{S}_{0} \rightarrow \mathrm{S}_{1}$ & 369 & 0.0340 & $n_{H-1} \rightarrow \pi_{L}^{*}$ & 66 & \\
\hline $\mathrm{S}_{0} \rightarrow \mathrm{S}_{2}$ & 363 & 0.4733 & $\pi_{H} \rightarrow \pi_{L}^{*}$ & 89 & 382 \\
\hline $\mathrm{S}_{0} \rightarrow \mathrm{S}_{3}$ & 331 & 0.0085 & $\pi_{H-2} \rightarrow \pi_{L}^{*}$ & 91 & \\
\hline $\mathrm{S}_{0} \rightarrow \mathrm{S}_{4}$ & 327 & 0.0143 & $\pi_{H-3} \rightarrow \pi_{L}^{*}$ & 71 & \\
\hline $\mathrm{S}_{0} \rightarrow \mathrm{S}_{5}$ & 323 & 0.0017 & $n_{H-5} \rightarrow \pi_{L}^{*}$ & 28 & \\
\hline $\mathrm{S}_{0} \rightarrow \mathrm{S}_{6}$ & 314 & 0.0023 & $n_{H-1} \rightarrow \pi_{L+1}^{*}$ & 48 & \\
\hline $\mathrm{S}_{0} \rightarrow \mathrm{S}_{7}$ & 304 & 0.0032 & $n_{H-4} \rightarrow \pi_{L}^{*}$ & 60 & \\
\hline $\mathrm{S}_{0} \rightarrow \mathrm{S}_{8}$ & 289 & 0.0016 & $\pi_{H} \rightarrow \pi_{L+1}^{*}$ & 93 & \\
\hline $\mathrm{S}_{0} \rightarrow \mathrm{S}_{9}$ & 277 & 0.0197 & $n_{H-6} \rightarrow \pi_{L+1}^{*}$ & 36 & \\
\hline $\mathrm{S}_{0} \rightarrow \mathrm{S}_{10}$ & 275 & 0.0120 & $\pi_{H-7} \rightarrow \pi_{L}^{*}$ & 54 & \\
\hline $\mathrm{S}_{0} \rightarrow \mathrm{S}_{11}$ & 274 & 0.0071 & $n_{H-4} \rightarrow \pi_{L+1}^{*}$ & 33 & \\
\hline $\mathrm{S}_{0} \rightarrow \mathrm{S}_{12}$ & 271 & 0.0384 & $n_{H-6} \rightarrow \pi_{L}^{*}$ & 33 & \\
\hline $\mathrm{S}_{0} \rightarrow \mathrm{S}_{13}$ & 270 & 0.1053 & $\pi_{H} \rightarrow \pi_{L+2}^{*}$ & 56 & 271 \\
\hline $\mathrm{S}_{0} \rightarrow \mathrm{S}_{14}$ & 267 & 0.0239 & $n_{H-11} \rightarrow \pi_{L}^{*}$ & 39 & \\
\hline $\mathrm{S}_{0} \rightarrow \mathrm{S}_{15}$ & 263 & 0.0180 & $n_{H-1} \rightarrow \pi_{L+3}^{*}$ & 41 & \\
\hline $\mathrm{S}_{0} \rightarrow \mathrm{S}_{16}$ & 258 & 0.2946 & $\pi_{H-2} \rightarrow \pi_{L+1}^{*}$ & 63 & \\
\hline $\mathrm{S}_{0} \rightarrow \mathrm{S}_{17}$ & 251 & 0.0476 & $n_{H-1} \rightarrow \pi_{L+2}^{*}$ & 68 & \\
\hline $\mathrm{S}_{0} \rightarrow \mathrm{S}_{18}$ & 250 & 0.0054 & $n_{H-8} \rightarrow \pi_{L}^{*}$ & 82 & \\
\hline $\mathrm{S}_{0} \rightarrow \mathrm{S}_{19}$ & 249 & 0.0122 & $\pi_{H} \rightarrow \pi_{L+3}^{*}$ & 89 & \\
\hline $\mathrm{S}_{0} \rightarrow \mathrm{S}_{20}$ & 247 & 0.0145 & $\pi_{H-3} \rightarrow \pi_{L+1}^{*}$ & 64 & \\
\hline $\mathrm{S}_{0} \rightarrow \mathrm{S}_{21}$ & 246 & 0.0497 & $\pi_{H-9} \rightarrow \pi_{L}^{*}$ & 42 & \\
\hline $\mathrm{S}_{0} \rightarrow \mathrm{S}_{22}$ & 244 & 0.3400 & $\pi_{H-3} \rightarrow \pi_{L+2}^{*}$ & 36 & \\
\hline $\mathrm{S}_{0} \rightarrow \mathrm{S}_{23}$ & 243 & 0.0373 & $n_{H-5} \rightarrow \pi_{L+1}^{*}$ & 44 & \\
\hline $\mathrm{S}_{0} \rightarrow \mathrm{S}_{24}$ & 242 & 0.1411 & $\pi_{H-2} \rightarrow \pi_{L+3}^{*}$ & 44 & \\
\hline $\mathrm{S}_{0} \rightarrow \mathrm{S}_{25}$ & 240 & 0.0247 & $\pi_{H-2} \rightarrow \pi_{L+2}^{*}$ & 80 & \\
\hline & & & & & \\
\hline
\end{tabular}




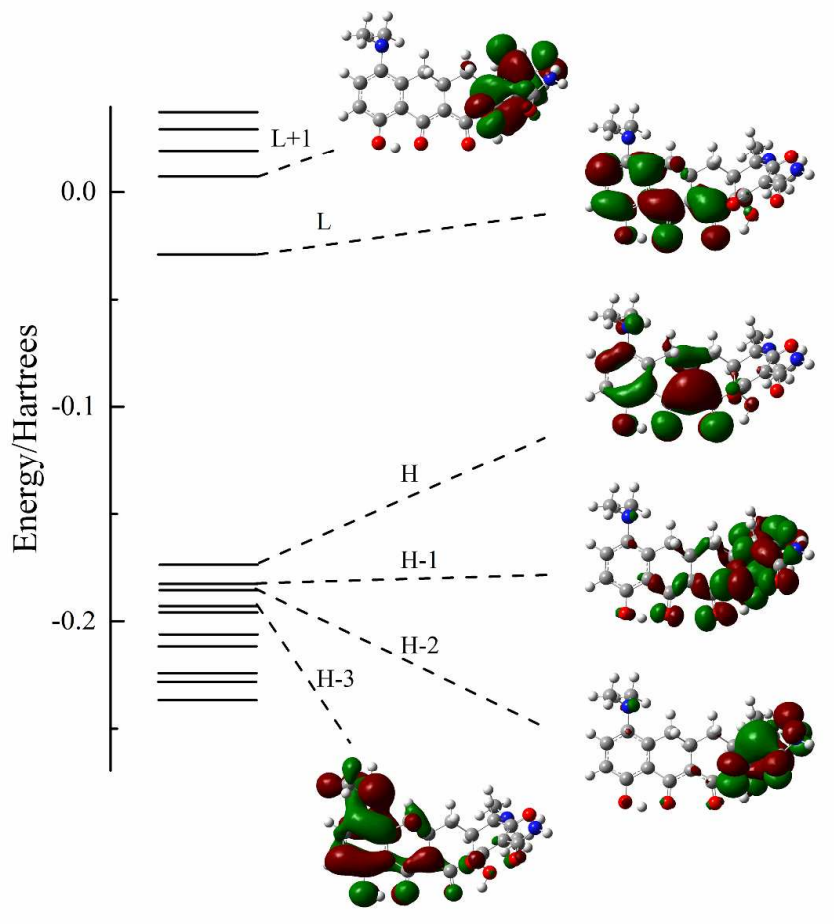

Figure S9. Molecular orbital energies and isodensity plots of $\mathrm{MC}^{2-}$ obtained by the B3LYP/6-31G(d) model.

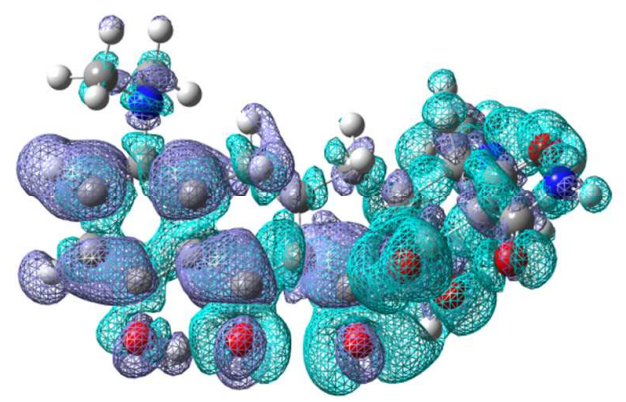

Figure S10. Effect of the $S_{0} \rightarrow S_{1}$ transition on the electron density of $\mathrm{MC}^{2-}$ (increase and decrease of electron densities are represented by violet and cyan, respectively). 


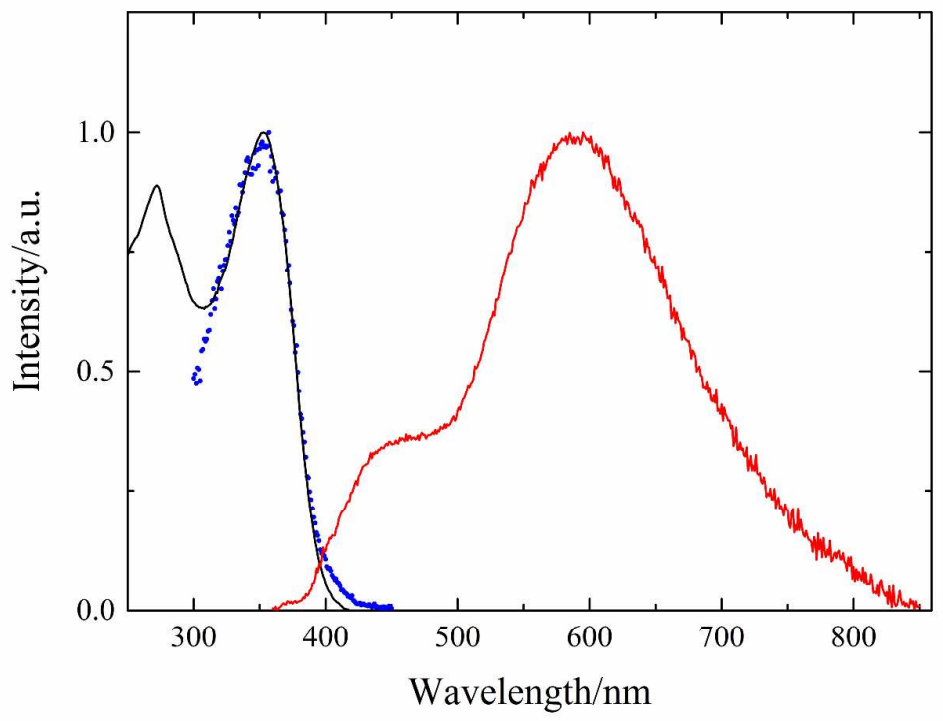

Figure S11. Characterization of $\mathrm{MCH}_{3}{ }^{+}$at $\mathrm{pH}$ 3.9: absorption spectrum (black), excitation spectrum (blue, emission at $590 \mathrm{~nm}$ ) and normalized emission spectrum (red, excitation at $350 \mathrm{~nm}$ ).

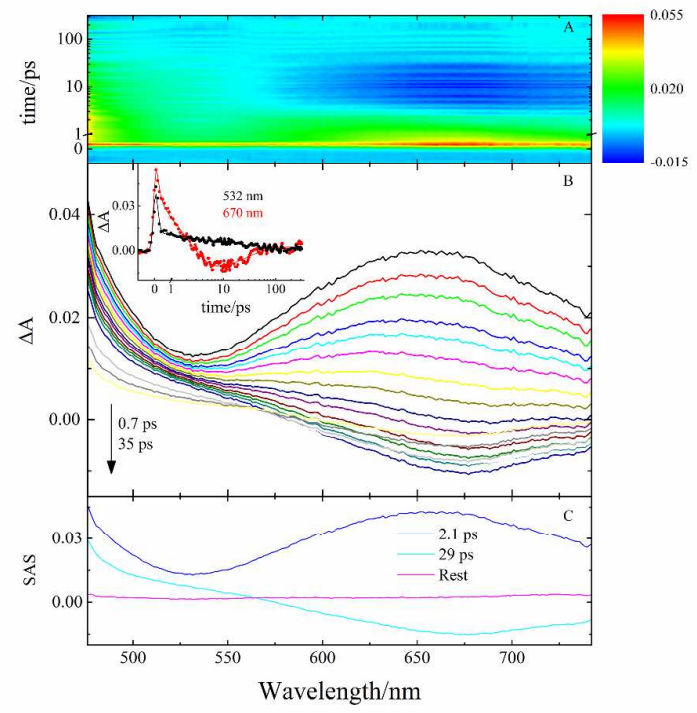

Figure S12. Pump-probe absorption $\left(\lambda_{\mathrm{exc}}=400 \mathrm{~nm}\right)$ of $\mathrm{MCH}_{3}{ }^{+}$in water at $\mathrm{pH}$ 3.9: A) contour plot of the experimental data, B) time resolved absorption spectra recorded at different delays after the laser pulse. Inset: decay kinetics recorded at meaningful wavelengths together with the corresponding fitting traces and C) Species Associated Spectra (SAS) of the decay components obtained by Target Analysis. 

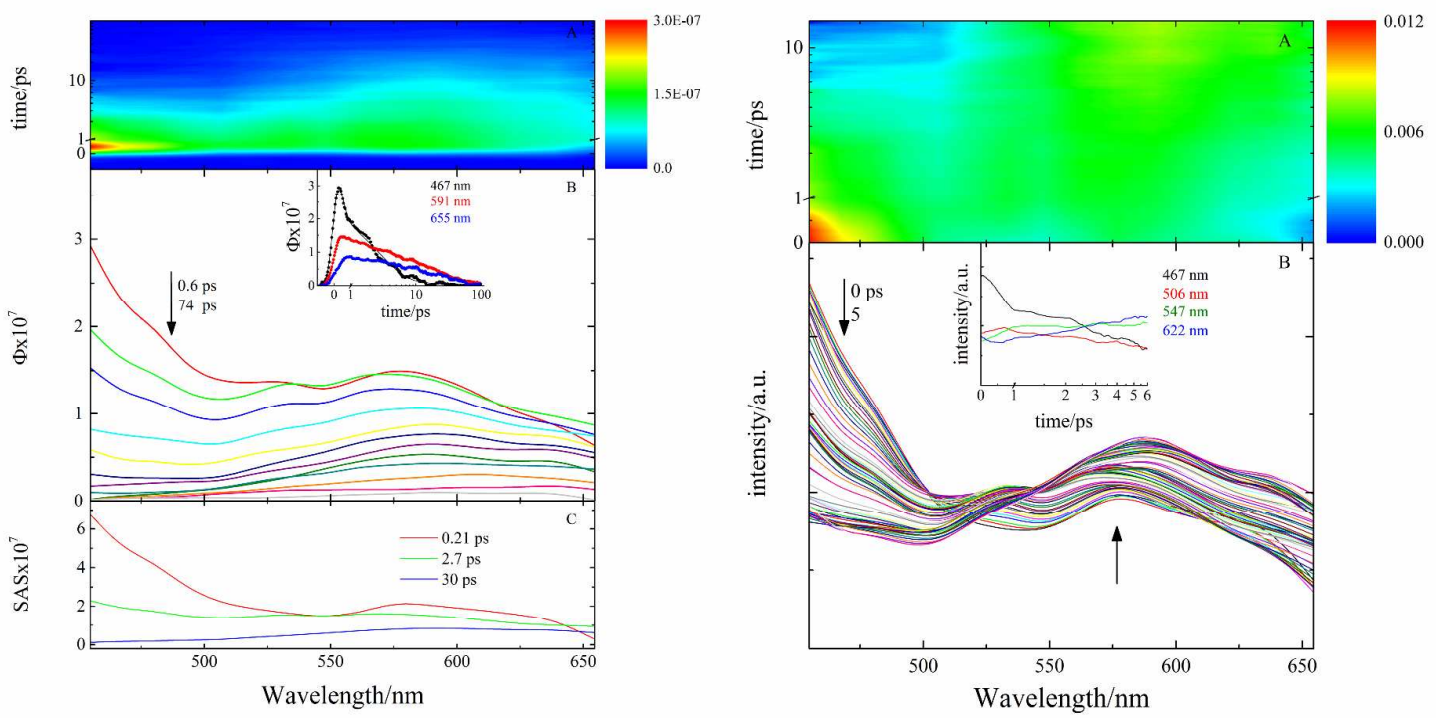

Figure S13. Fluorescence up-conversion $\left(\lambda_{\text {exc }}=400 \mathrm{~nm}\right)$ of $\mathrm{MCH}_{3}{ }^{+}$in water solution at $\mathrm{pH}$ 3.9. Left graph: A) contour plot of the experimental data, B) time resolved emission spectra recorded between 0.6 and $74 \mathrm{ps}$ after the laser pulse. Inset: decay kinetics recorded at meaningful wavelengths together with the corresponding fitting traces and C) SAS of the decay components obtained by Target Analysis. Right graph: A) contour plot of the TRANES and B) their spectral evolution recorded between 0 and $14 \mathrm{ps}$ after the laser pulse. Inset: time-profile of the TRANES.

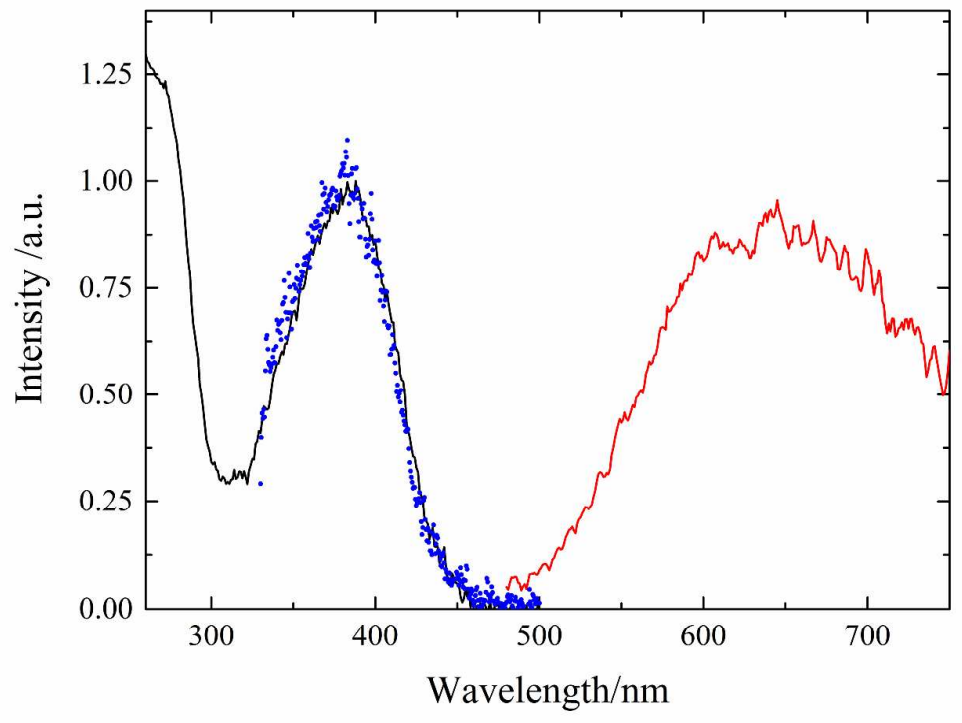

Figure S14. Absorption (black line), excitation (blue dots, emission at $645 \mathrm{~nm}$ ) and emission (red line, excitation at $385 \mathrm{~nm}$ ) spectra of MC in DMSO. 


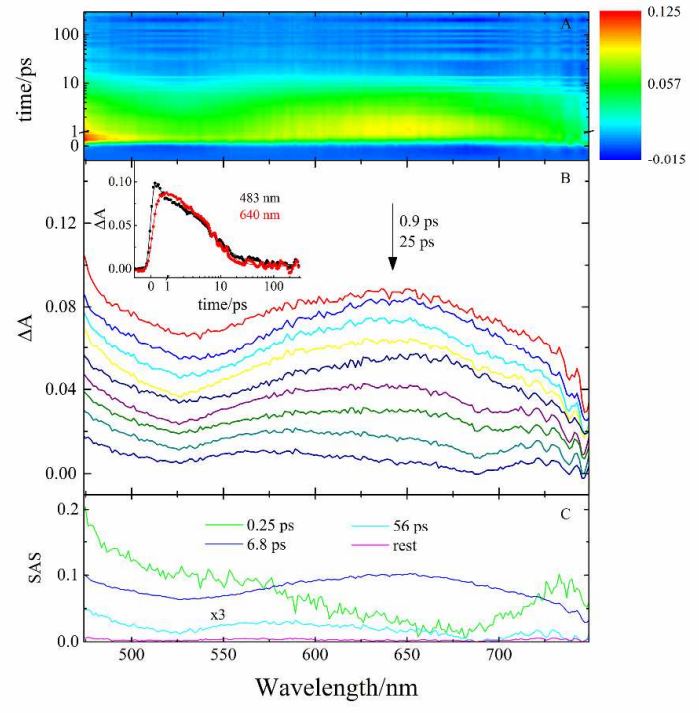

Figure S15. Pump-probe absorption $\left(\lambda_{\mathrm{exc}}=400 \mathrm{~nm}\right)$ of $\mathrm{MC}$ in $\mathrm{MeCN}$ : A) contour plot of the experimental data, B) time resolved absorption spectra recorded at different delays after the laser pulse. Inset: decay kinetics recorded at meaningful wavelengths together with the corresponding fitting traces and C) Species Associated Spectra (SAS) of the decay components obtained by Target Analysis.

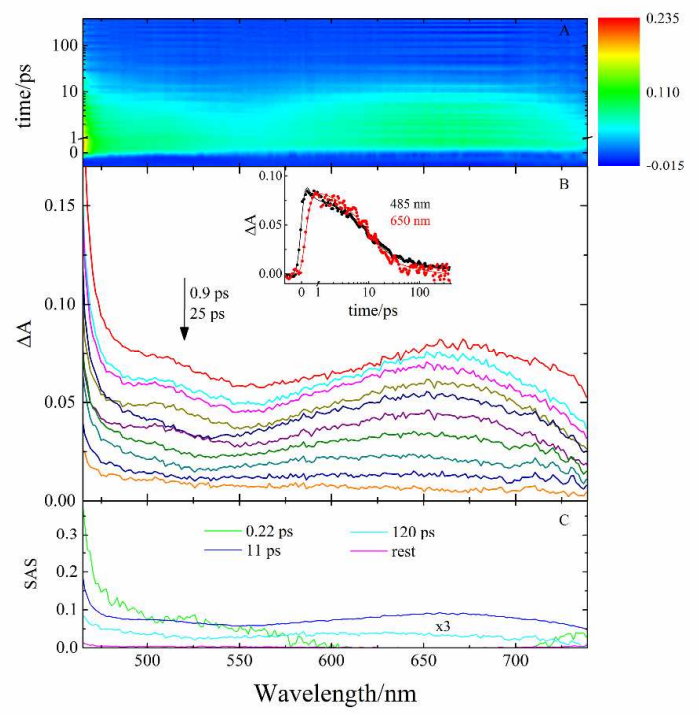

Figure S16. Pump-probe absorption $\left(\lambda_{\text {exc }}=400 \mathrm{~nm}\right)$ of MC in DMSO: A) contour plot of the experimental data, B) time resolved absorption spectra recorded at different delays after the laser pulse. Inset: decay kinetics recorded at meaningful wavelengths together with the corresponding fitting traces and C) Species Associated Spectra (SAS) of the decay components obtained by Target Analysis. 


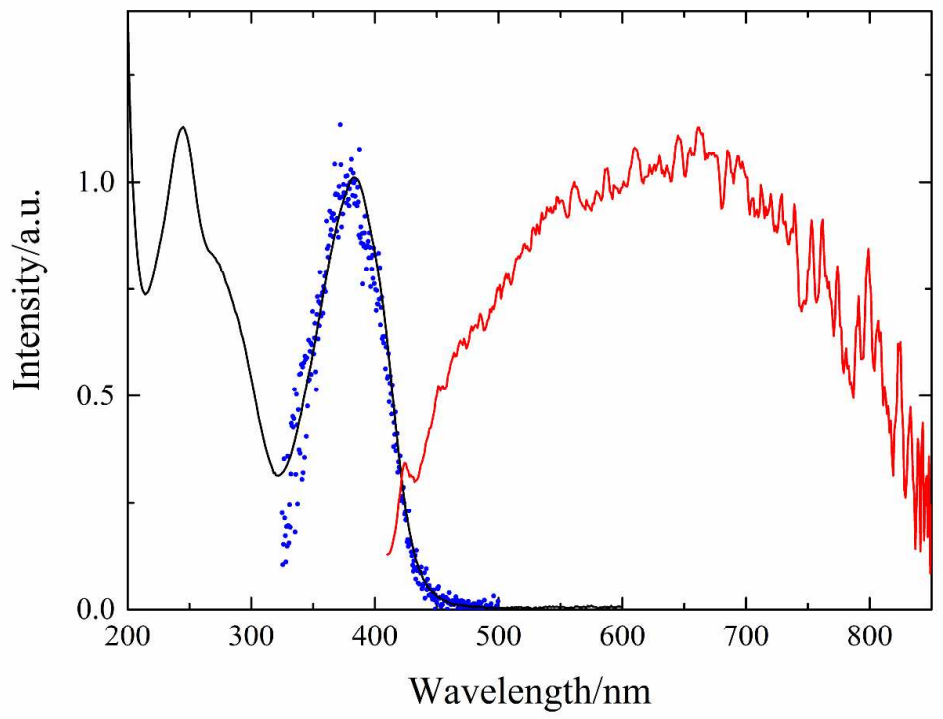

Figure S17. Characterization of $\mathrm{MC}^{2-}$ at $\mathrm{pH}$ 11.3: absorption spectrum (black line), excitation spectrum (blue dots, emission at $680 \mathrm{~nm}$ ) and normalized emission spectrum (red line, excitation at $385 \mathrm{~nm}$ ).

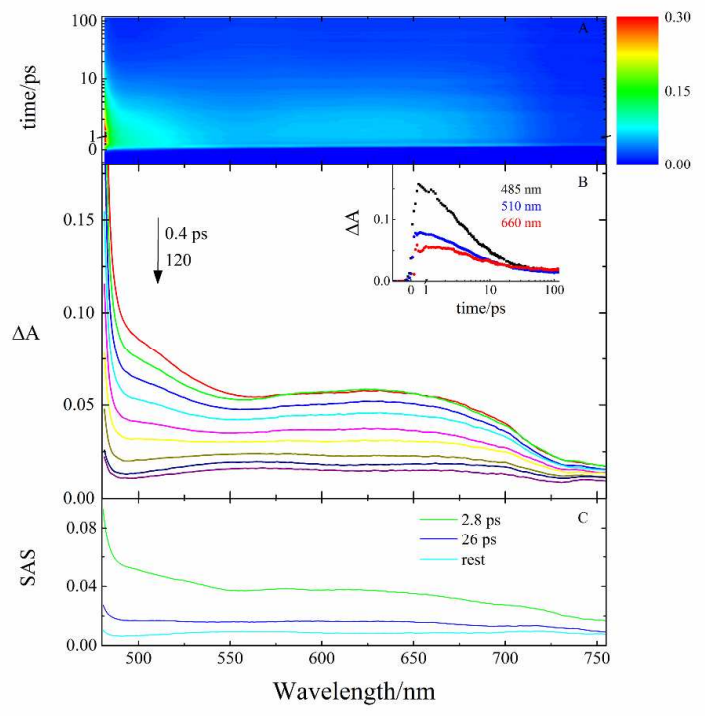

Figure S18. Pump-probe absorption $\left(\lambda_{\mathrm{exc}}=400 \mathrm{~nm}\right)$ of $\mathrm{MCH}^{-}$in water at $\mathrm{pH}$ 9.0: A) contour plot of the experimental data, B) time resolved absorption spectra recorded at different delays after the laser pulse. Inset: decay kinetics recorded at meaningful wavelengths together with the corresponding fitting traces and C) Species Associated Spectra (SAS) of the decay components obtained by Target Analysis. 


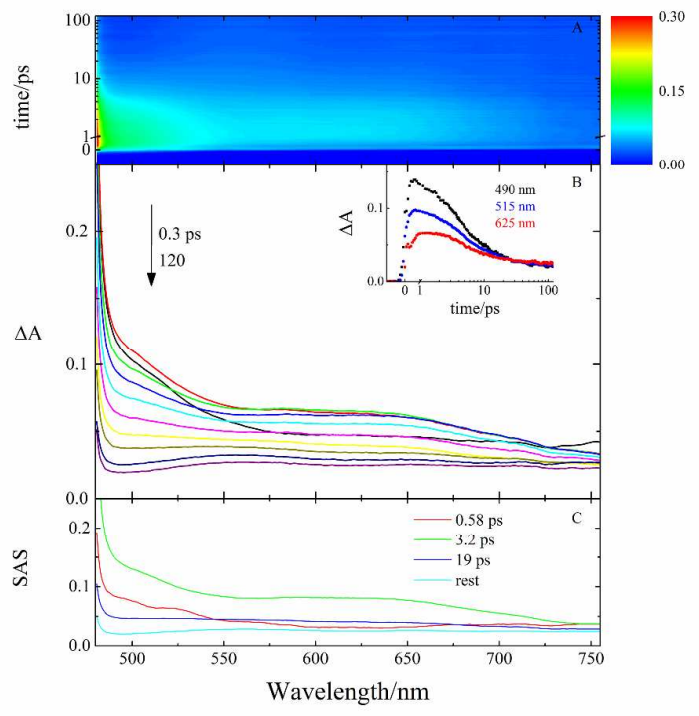

Figure S19. Pump-probe absorption $\left(\lambda_{\text {exc }}=400 \mathrm{~nm}\right)$ of $\mathrm{MCH}^{2-}$ in water at $\mathrm{pH}$ 11.3: A) contour plot of the experimental data, B) time resolved absorption spectra recorded at different delays after the laser pulse. Inset: decay kinetics recorded at meaningful wavelengths together with the corresponding fitting traces and C) Species Associated Spectra (SAS) of the decay components obtained by Target Analysis.
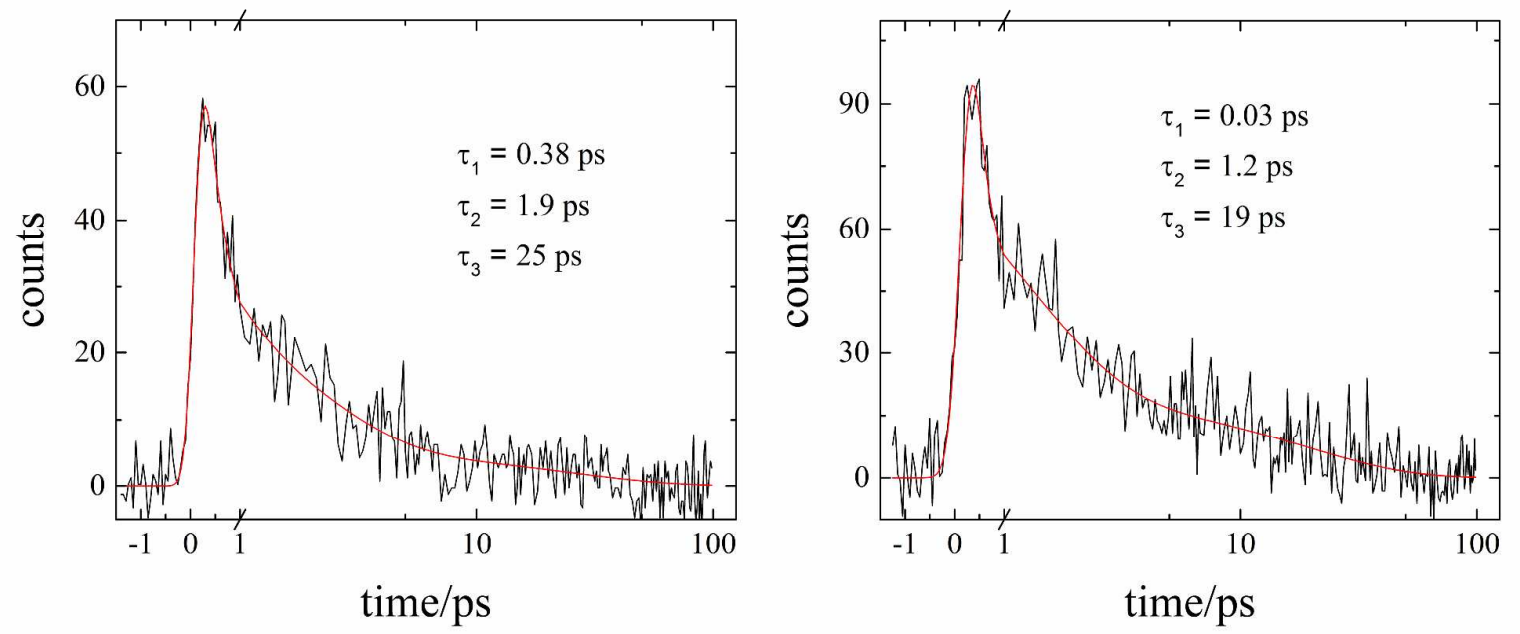

Figure S20. Fluorescence up-conversion $\left(\lambda_{\mathrm{exc}}=400 \mathrm{~nm}\right.$ ) of $\mathrm{MCH}^{-}$and $\mathrm{MC}^{2-}$ in water solution at $\mathrm{pH} 9.0$ (left) and $\mathrm{pH} 11.3$ (right), respectively. Decay kinetics recorded at meaningful wavelengths together with the corresponding fitting traces. 


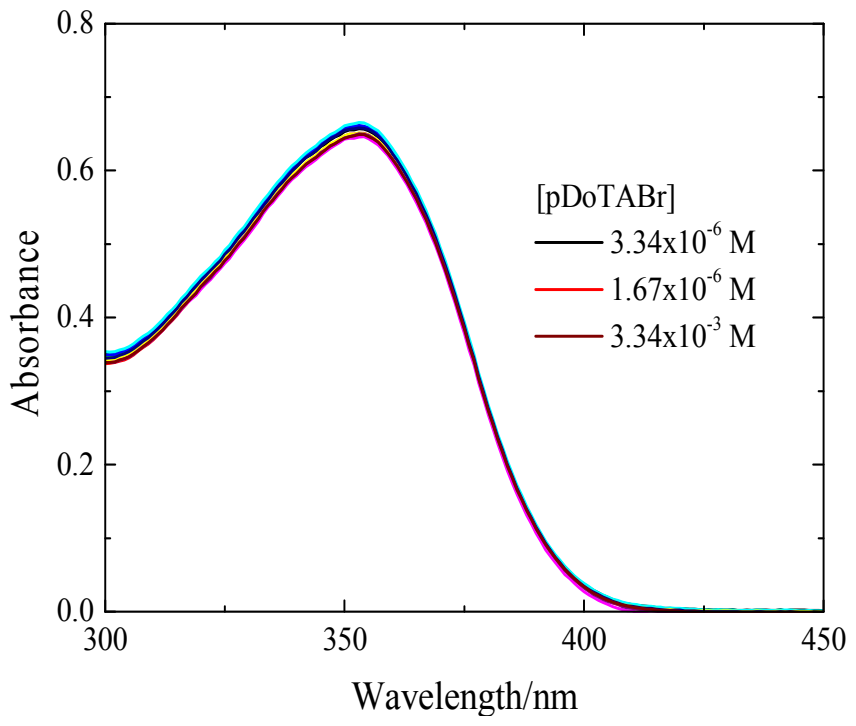

Figure S21. Absorption spectra of $\mathrm{MCH}_{2}{ }^{2+}$ in aqueous solution at $\mathrm{pH} 2.3$ alone and in the presence of increasing amount of $p$ DoTABr up to $3.34 \mathrm{mM}$.

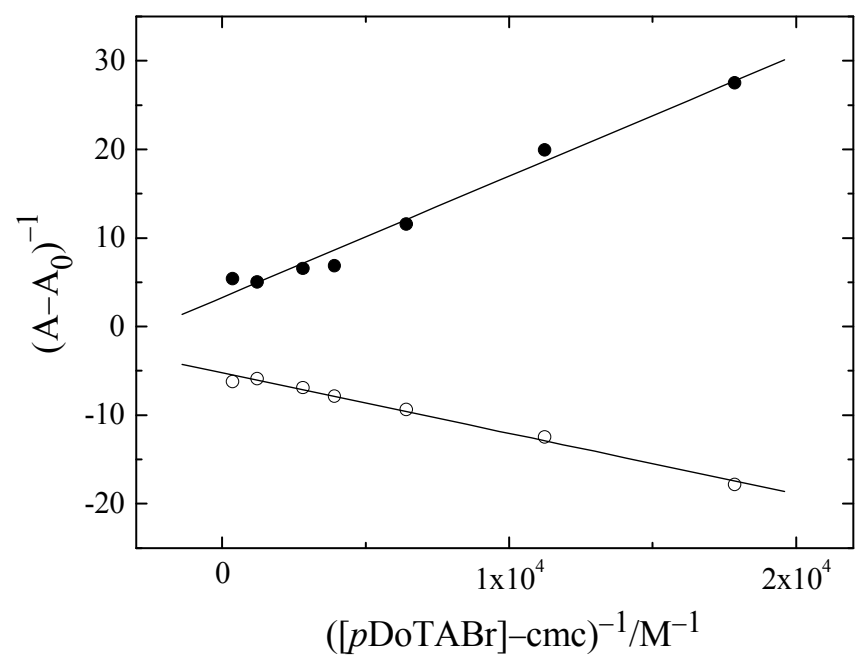

Figure S22. $\left(\mathrm{A}-\mathrm{A}_{0}\right)^{-1}$ vs. $([\mathrm{pDoTABr}]-\mathrm{cmc})^{-1}$ (eq. 1) of $\mathrm{MCH}_{2}{ }^{ \pm}$in aqueous solution at $\mathrm{pH} 6.4$ calculated at 310 (open circles) and 385 (full circles) $\mathrm{nm}$. 


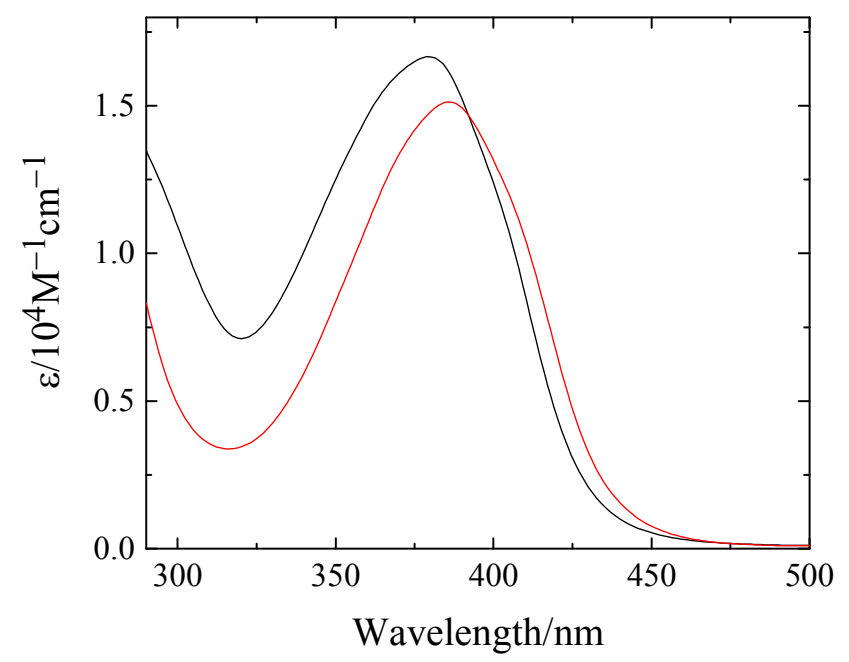

Figure S23. Quantitative absorption spectra of $\mathrm{MCH}^{-}$(black line) and its complex with $p$ DoTABr (red line) obtained by SVD and Global Analysis $\left(\mathrm{K}_{\mathrm{ass}}=1.2 \times 10^{5} \mathrm{M}\right)$.

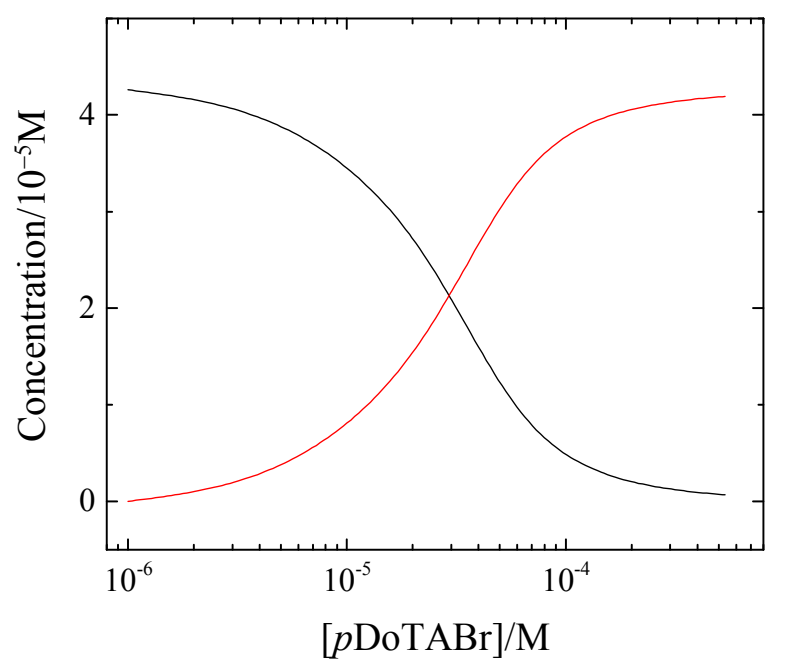

Figure S24. Concentration profile of $\mathrm{MCH}^{-}$(black line) and its complex with $p$ DoTABr (red line) obtained by SVD and Global Analysis. 


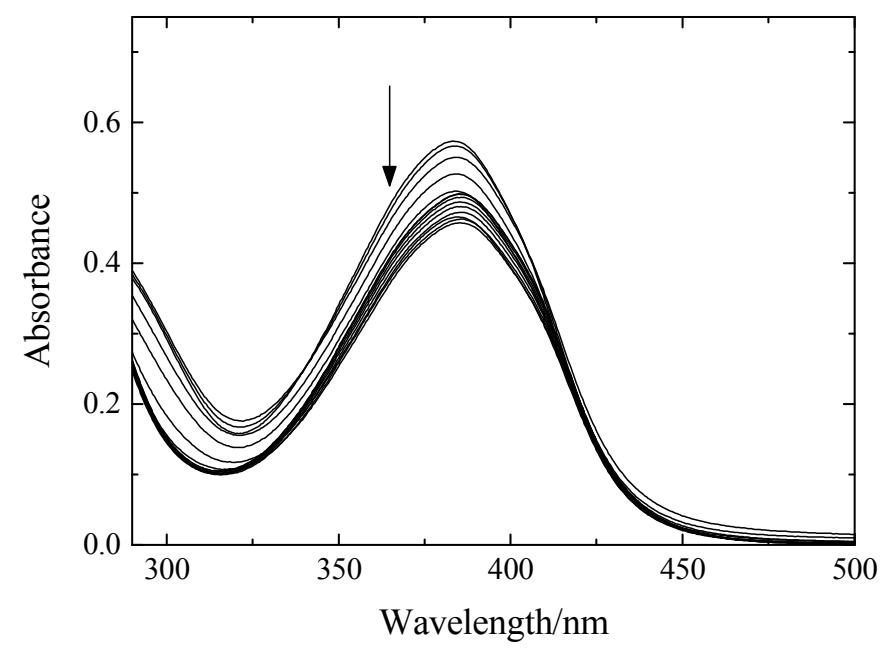

Figure S25. Absorption spectra of $\mathrm{MC}^{2-}$ in aqueous solution at $\mathrm{pH} 11.3$ alone and in the presence of increasing amount of $p$ DoTABr up to $4.1 \mathrm{mM}$.

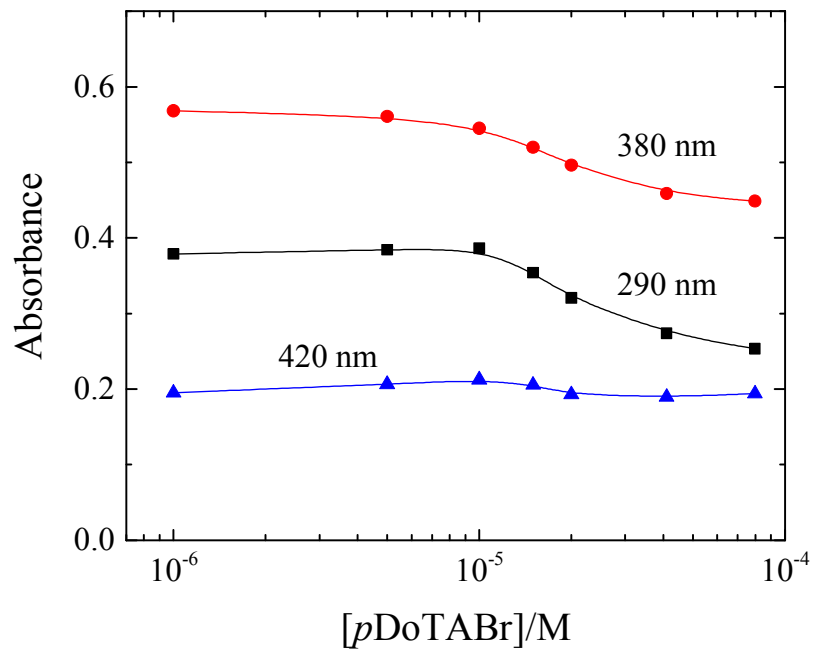

Figure S26. Dependence of the absorbance of $\mathrm{MC}^{2-}$ upon $p$ DoTABr concentration at three significant wavelengths. 


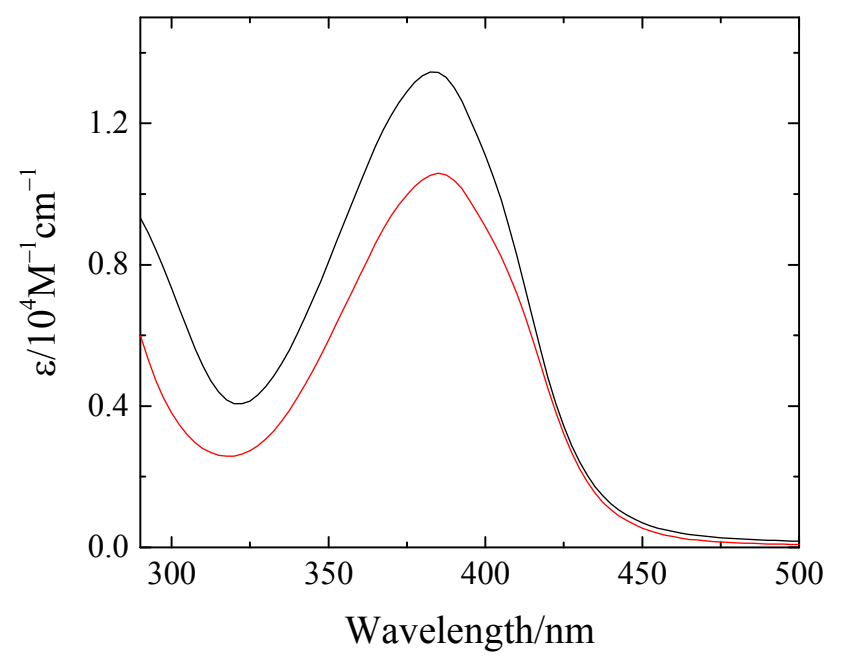

Figure S27. Quantitative absorption spectra of $\mathrm{MC}^{2-}$ (black line) and its complex with $p$ DoTABr (red line) obtained by SVD and Global Analysis $\left(\mathrm{K}_{\mathrm{ass}}=2.3 \times 10^{6} \mathrm{M}\right)$.

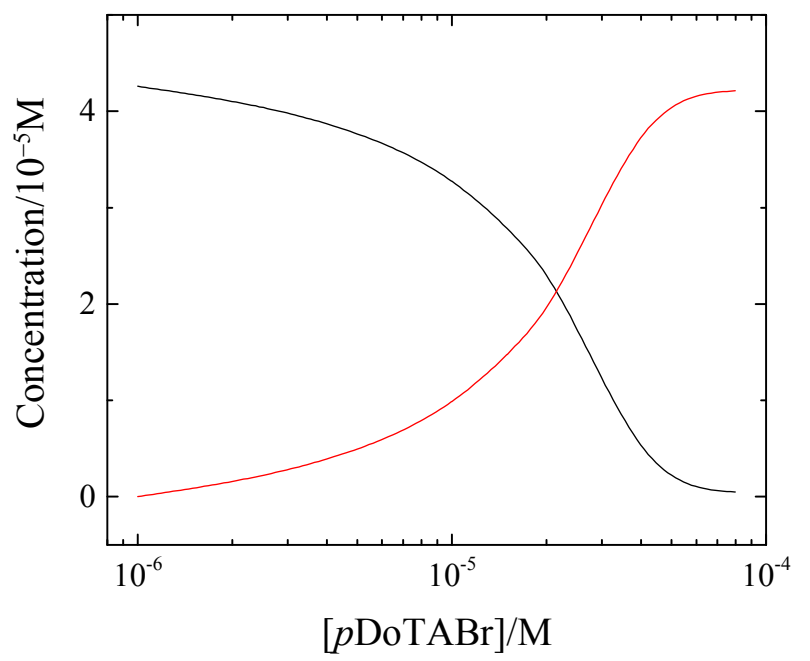

Figure S28. Concentration profile of $\mathrm{MC}^{2-}$ (black line) and its complex with $p \mathrm{DoTABr}$ (red line) obtained by SVD and Global Analysis. 


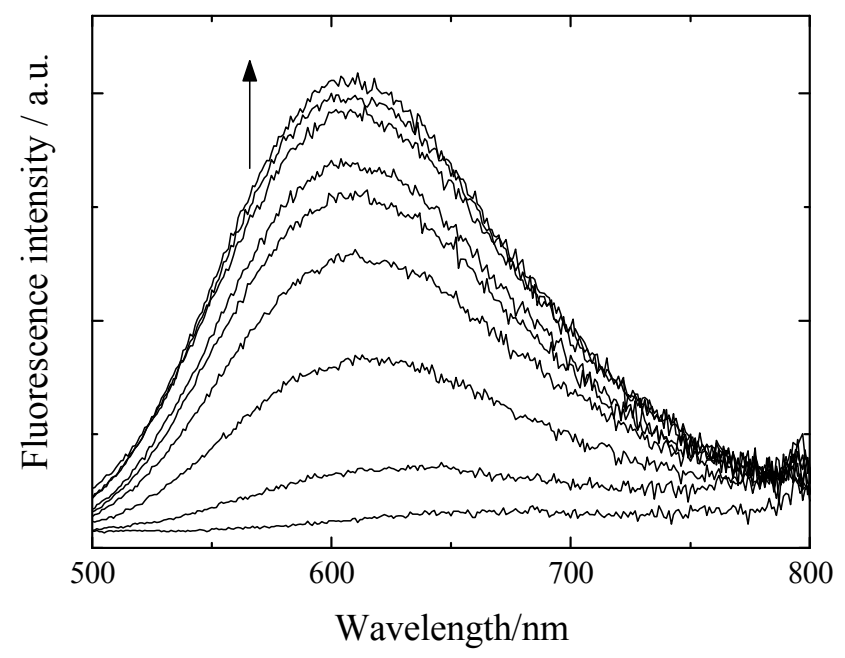

Figure S29. Fluorescence spectra $\left(\lambda_{\mathrm{exc}}=417 \mathrm{~nm}\right)$ of $\mathrm{MC}^{2-}$ in aqueous solution at $\mathrm{pH} 11.3$ alone and in the presence of increasing amount of $p$ DoTABr up to $1.8 \mathrm{mM}$. 